\title{
Innovations and Emerging Therapies in Glaucoma
}

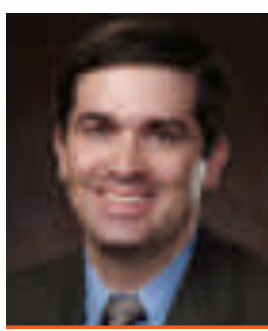

Chris Wroten, O.D., Dipl. ABO, is a graduate of Southern College of Optometry (SCO) and a partner and Chief Operating Officer for the Bond-Wroten Eye Clinics. Dr. Wroten has participated in clinical research, authored clinical case reports and eyecare articles, lectured as a continuing education speaker, and presented educational posters and workshops at regional and national optometric conferences. In addition to primary eye care, his special areas of interest lie in the treatment and management of ocular disease and contact lenses.

$\mathrm{I}$ t's unclear when the progressive optic neuropathy that is glaucoma was first discovered, but it has been described in medical writings since ancient times. Hippocrates wrote of a blindness in the elderly that he called "glaykoseis." Much later, the English ophthalmologist Richard Banister first discovered a correlation with high intraocular pressure (IOP). The invention of the ophthalmoscope by Hermann von Helmholtz in the mid-1800's allowed the in vivo visualization of glaucomatous changes to the optic nerve for the first time, and in 1862 Franciscus Donders coined the term "Glaukoma simplex" to describe blindness resulting from elevated IOP. Shortly thereafter, the invention of the tonometer, the development of perimetry, and the use of cocaine as an anesthetic all further advanced the diagnosis of glaucoma.

The first therapies for glaucoma were actually surgical procedures; Friedrich von Graefe performed the first iridectomy in 1856. Since then, trabeculectomy, trabeculoplasty, and drainage tube implants, among other procedures, as well as micro-invasive glaucoma surgeries (MIGS) most recently, have also been developed as effective treatment options. Perhaps somewhat surprisingly, pharmaceutical treatment of glaucoma did not begin until 1875 with the discovery of pilocarpine, and then advanced rather slowly over the next 120 years with the development of topical beta blockers, alpha agonists, and carbonic anhydrase inhibitors. The launch of topical prostaglandin analogs around the turn of the $21^{\text {st }}$ century marked another milestone in glaucoma treatment by simultaneously reducing the dosage frequency and improving the ocular hypotensive effect. ${ }^{1}$

Yet the prevalence of glaucoma, which is often described as "the sneak thief of sight," continues to increase. In fact, by the year 2020, the worldwide prevalence of glaucoma in people 40-80 years of age is projected to be 76 million, with a further increase to 112 million by 2040, and this will disproportionately impact populations in Africa and Asia (Fig. 1). ${ }^{2}$

Globally, it is estimated that 4.5 million patients are blind due to glaucoma, ${ }^{2}$ making it the second-leading cause of blindness worldwide according to the World Health Organization. Despite expanded patient education, new diagnostic technologies, improved understanding of its pathophysiology, greater choices in pharmaceutical therapies, and improvements in surgical options and outcomes, the number of cases of blindness from glaucoma is expected to increase to 11.2 million by $2020 .{ }^{3}$ Furthermore, even in developed countries, it's estimated that up to $50 \%$ of affected patients are not even aware they have glaucoma due to its insidious nature, and this percentage is as high as $90 \%$ in underdeveloped countries. ${ }^{4}$ Given these staggering numbers, research continues in the hope of one day discovering a cure for this disease, while pharmaceutical and therapeutic options continue to evolve and improve. As doctors of optometry, we have played a crucial role in saving the sight of countless patients with glaucoma in recent decades. Let's take a brief look at some emerging treatments for glaucoma that may further enhance quality of life and reduce morbidity for our patients with this disease.

\section{PHARMACEUTICAL AGENTS}

While it had previously been over two decades since the introduction of a truly novel class of glaucoma medication, in December 2017, two new glaucoma agents with unique mechanisms of action were approved for use by the United States Food and Drug Administration. The first was latanoprostene bunod (Vyzulta" ${ }^{\text {Tn }}$, Bausch + Lomb, Rochester, NY), a once-daily topical medication that breaks down into latanoprost acid, a well-established prostaglandin analog that remodels the ciliary muscle's extracellular matrix to enhance uveoscleral outflow of aqueous, and also a novel donor of nitric oxide, which further acts directly on the trabecular meshwork to relax smooth muscle and further enhance aqueous outflow (Fig. 2).5,6 


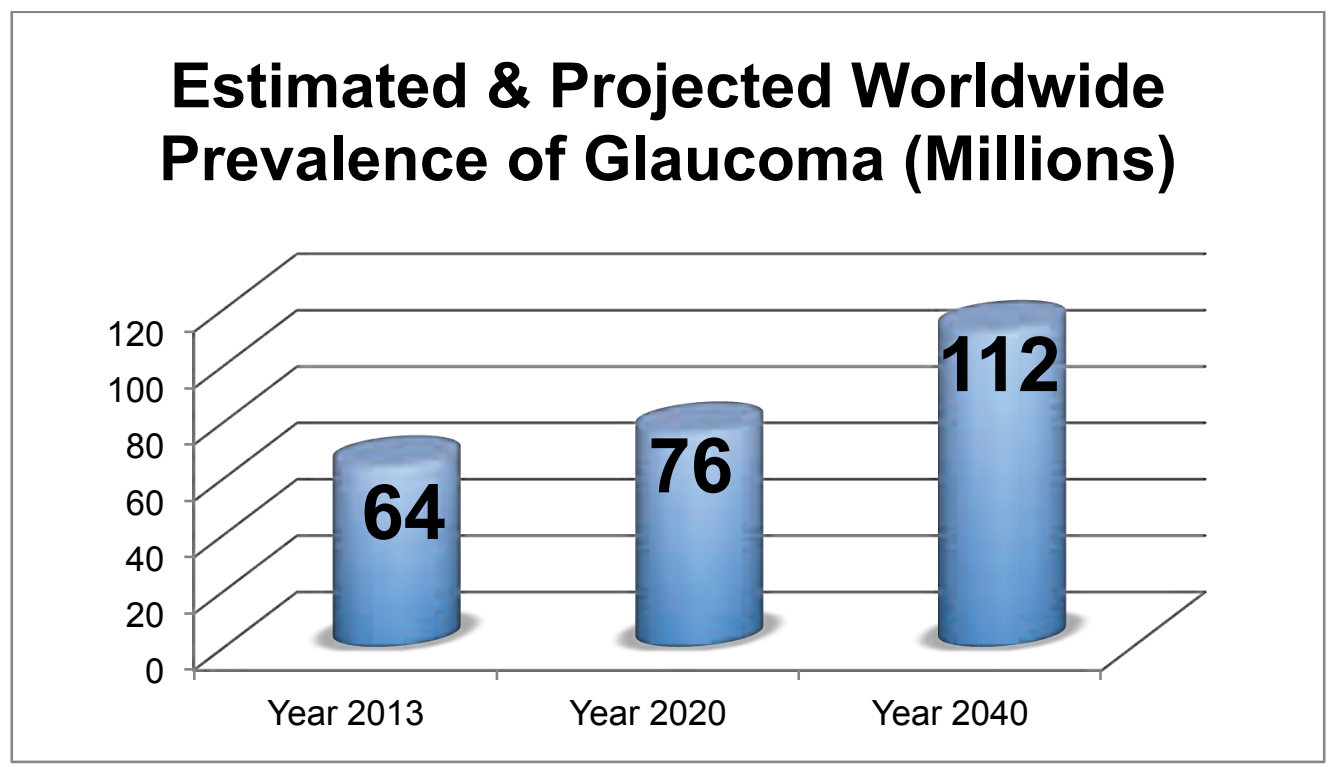

Figure 2: The dual mechanism of action of latanoprostene bunod (Vyzulta ${ }^{\mathrm{TM}}$ ) includes the traditional prostaglandin analog effect of enhanced uveoscleral outflow via latanoprost acid, as well as its effect as a donor of nitric oxide acting directly on the trabecular meshwork and Schlemm's canal to further facilitate aqueous outflow. (See Ref. 6)

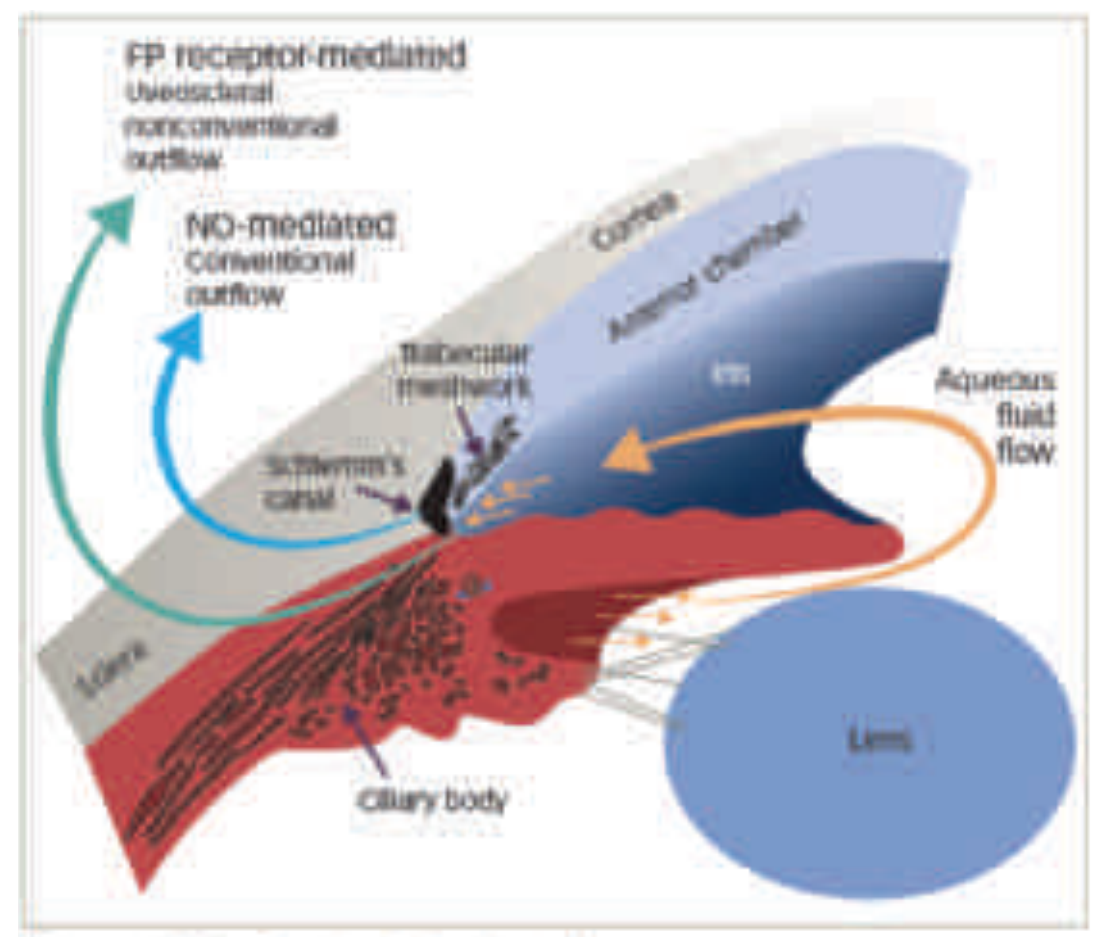

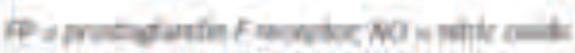


The second newly approved pharmaceutical for glaucoma, netarsudil (Rhopressa ${ }^{\mathrm{TN}}$, Aerie Pharmaceuticals, Durham, NC), represents an entirely new class of medications called Rho-kinase (ROCK) inhibitors. ROCK inhibitors relax smooth muscle in the trabecular meshwork to increase aqueous outflow, while also lowering episcleral venous pressure and further facilitating outflow, both of which are unique mechanisms of action that are not shared with any other currently available glaucoma medications. Netarsudil also includes a Nor-epinephrine Transport (NET) inhibitor that suppresses aqueous production, to give a triple mechanism of action (Fig. 3).?

Figure 3: The triple mechanism of action of netarsudil (Rhopress $\mathrm{A}^{\mathrm{TM}}$ ) includes relaxation of the trabecular meshwork (TM) tissue, inhibition of aqueous production, and lowering of the episcleral venous pressure (EVP). (Source: Aerie Pharmaceuticals)

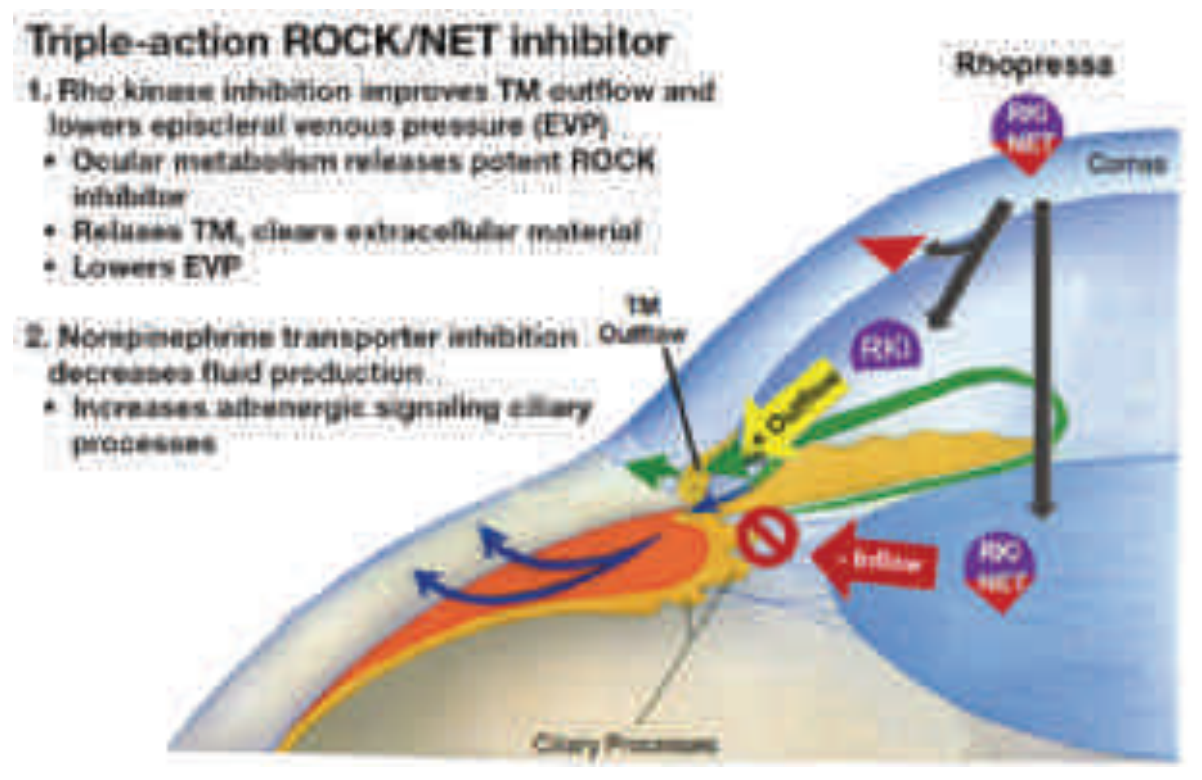

Combined formulations of ROCK- and NET-inhibitors, paired with a prostaglandin analog to also enhance uveoscleral outflow, thus offering a quadruple mechanism of action, are in development and may be commercially available within the next year.

Research aimed at improving the delivery of existing ocular hypotensive pharmaceutical agents continues, and various companies have reported early success in trials for glaucoma drug-eluting contact lenses, conjunctival fornix-based inserts, punctal plugs, and intracanalicular inserts. ${ }^{8-10}$ Formulations of currently available pharmaceutical agents for glaucoma in injectable form for use in and around the eye are also being investigated, as well as the 3-D printing of existing pharmaceuticals and their nano-sized counterparts to exponentially enhance efficacy and reduce dosage frequency while simultaneously reducing drug concentrations.

Another completely novel molecule that shows potential for use in glaucoma is aminoguanidine, which was originally developed for treating diabetic nephropathy. It is an inhibitor of nitric oxide synthase, an enzyme present in elevated levels in the optic nerves of patients with glaucoma. In an early study on lab rats with chronic, moderately elevated IOP, whose drinking water was laced with this medication, animals treated with aminoguanidine lost $10 \%$ of their retinal ganglion cells, while an untreated group lost $36 \%$ of their retinal ganglion cells. ${ }^{11}$ The key takeaway here was that aminoguanidine had no effect on IOP whatsoever, yet still seemed to offer some form of neuroprotection. To date, there have been no human clinical trials, and there is some debate within the research community regarding the role that nitric oxide synthase may or may not play in glaucoma.

These compounds represent just a small sampling of what's in the research and development pipeline, as we continue to hold out hope for more affordable and more effective glaucoma medications. 


\section{THERAPEUTIC PROCEDURES}

Therapeutic procedures for glaucoma also continue to be investigated, including an intraocular lens implant that could continuously monitor a "pseudo"-intraocular pressure, similar to the Triggerfish "Contact Lens. Additionally, a low-power, low-frequency, ultrasound device to treat glaucoma is being developed by two different companies. One of these devices, the Therapeutic Ultrasound for Glaucoma (TUG ${ }^{\mathrm{TM}}$ by Eye Sonix, Long Beach, CA, Fig. 4), is designed to trigger an inflammatory reaction in the anterior chamber and trabecular meshwork to enhance aqueous outflow, and an early trial reported a $20 \%$ reduction in IOP lasting for at least one year in $74 \%$ of patients with an elevated pre-treatment IOP. ${ }^{12}$

Figure 4: The Therapeutic Ultrasound for Glaucoma (TUG ${ }^{\mathrm{TM}}$ by Eye Sonix) is one of at least two ultrasound technologies being developed for glaucoma that are non-incisional procedures to enhance aqueous outflow. (Source: Eye Sonix)

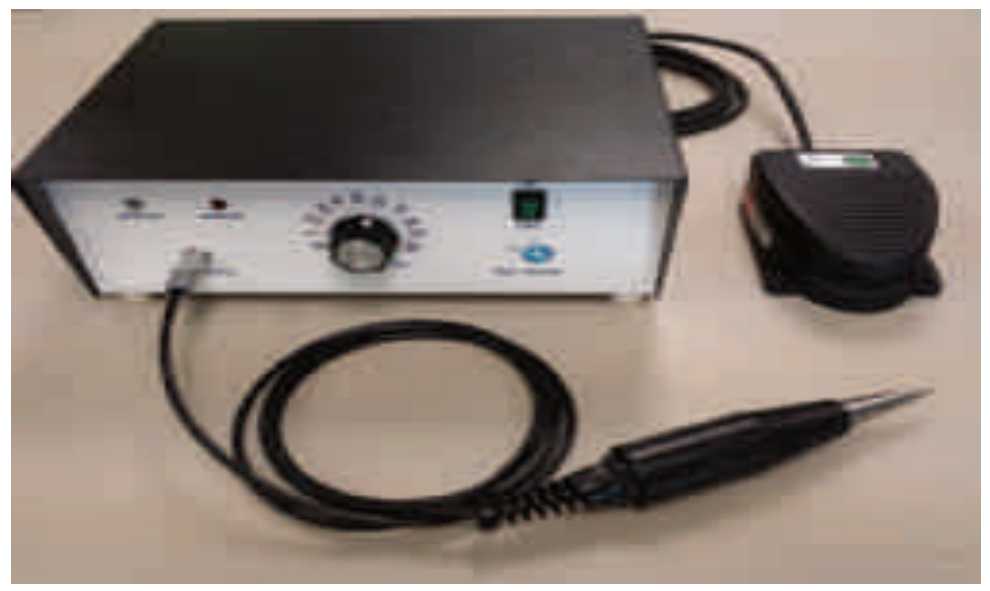

Figure 5: The Balance Goggles ${ }^{\mathrm{TM}}$ being developed by Berdahl would apply a small vacuum over the eyes of patients with glaucoma to normalize their IOP-ICP pressure differential. (Source: Equinox)

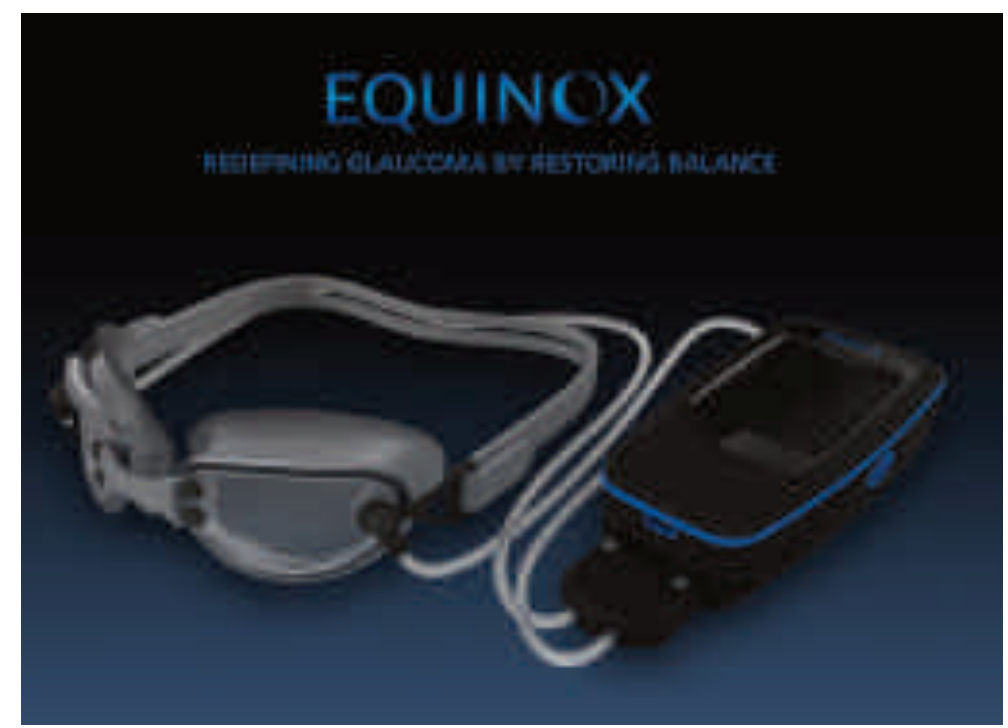


Lastly, Berdahl hypothesized that glaucoma is the result of an imbalance between a patient's intracranial pressure (ICP) and their IOP, and that when IOP is significantly greater than ICP, glaucomatous damage occurs as a result of this pressure differential and its effect on the optic nerve's metabolism. ${ }^{13} \mathrm{He}$ is developing Balance Goggles ${ }^{\mathrm{wn}}$ (Equinox, Sioux Falls, SD), which are similar to swim goggles with a small vacuum device attached, which a patient with glaucoma would wear at night to draw a small vacuum above the eyes and normalize the IOP-ICP pressure differential (Fig. 5). Human clinical trials of this novel concept are now underway, and it potentially represents the first treatment for glaucoma that is non-surgical and does not involve pharmaceuticals.

This is obviously just a small sampling of current innovations in the treatment of glaucoma. As the pipeline of new and emerging eye care treatments and technologies continues to expand for glaucoma and elsewhere within our profession, our job remains to responsibly vet each innovation, advocate to protect patients from those that don't meet established standards of care, and embrace and appropriately implement the new technologies and treatments that do to enhance our patients' quality of life and expand the scope of care we provide. As we strive to achieve these goals, and until a cure for glaucoma is discovered, hopefully the "sneak thief of sight" will continue to be caught red-handed and arrested more often than ever before.

\section{REFERENCES}

1. Grewe, R. The history of glaucoma. Klin Monbl Augenheilkd 1986 Feb; 188(2): 167-9.

2. Tham YC, Li X, Wong TY, Quigley HA, Aung T, Cheng CY. Global prevalence of glaucoma and projections of glaucoma burden through 2040: A systematic review and meta-analysis. Ophthalmology 2014 Nov; 121(11): 2081-90.

3. Quigley HA, Broman AT. The number of people with glaucoma worldwide in 2010 and 2020. Br J Ophthalmol 2006 Mar; 90:262-7.

4. Sommer A, Tielsch JM, Katz J, et al. Relationship between intraocular pressure and primary open angle glaucoma among white and black Americans. The Baltimore Eye Survey. Arch Ophthalmol 1991 Aug; 109(8): 1090-5.

5. Weinreb RN, Liebmann JM, Martin KR, Kaufman PL, Vittitow JL. Latanoprostene bunod $0.024 \%$ in subjects with open-angle glaucoma or ocular hypertension: pooled phase 3 study findings. $J$ Glaucoma 2018 Jan;27(1):7-15.

6. Weinreb RN, Realini T, Varma R. Latanoprostene bunod, a dualacting nitric oxide donating prostaglandin analog for lowering of intraocular pressure. US Ophthalmic Rev 2016;9(2):80-7.
7. Ren R, Li G, Le TD, Kopczynski C, Stamer WD, Gong H. Netarsudi increases outflow facility in human eyes through multiple mechanisms. Invest Ophthalmol Vis Sci. 2016 Nov 1;57(14):6197-209.

8. Lusthaus JA, Goldberg I. Emerging drugs to treat glaucoma: targeting prostaglandin $\mathrm{F}$ and $\mathrm{E}$ receptors. Expert Opin Emerg Drugs 2016;21(1):117-28.

9. Hui A, Willcox M. in vivo studies evaluating the use of contact lenses for drug delivery. Optom Vis Sci 2016 Apr;93(4):367-76.

10. Chen H. Recent developments in ocular drug delivery. J Drug Target 2015;23(7-8):597-604.

11. Neufeld AH, Sawada A, Becker B. Inhibition of nitric-oxide synthase 2 by aminoguanidine provides neuroprotection of retinal ganglion cells in a rat model of chronic glaucoma. Proc Natl Acad Sci USA 1999; 96 (17): 9944-8.

12. Schwartz D, Samples J, Korosteleva O. Therapeutic ultrasound for glaucoma: clinical use of a low-frequency low-power ultrasound device for lowering intraocular pressure. J Ther Ultrasound 2014; $2: 15$.

13. Berdahl J. The eye in space. US Ophthalmic Rev 2016; Sep 26, 2016; 9(2):76-7. 